prophylaxis. 2 patients (18\%) were started on prophylaxis and in 1 patient it was continued.

Conclusion The documentation of decision making regarding VTE prophylaxis in $\mathrm{RH}$ was initially poor. Following implementation of the assessment tool there has been improvement in documenting and discussion around VTE prophylaxis.

Data collection is still ongoing. The next step will be to assess if VTE prophylaxis prescriptions are reviewed, e.g. monitoring platelets, reviewing the duration and appropriateness of treatment.

\section{THE DEVELOPMENT OF AN ENHANCED NURSING HOME BEDS SERVICE PROVIDES AN ALTERNATIVE TO HOSPITALISATION FOR PEOPLE APPROACHING THE END OF THEIR LIVES}

Caroline McCluskey, Robert Smith. Derby Teaching Hospitals NHS Foundation Trust

\subsection{6/bmjspcare-2018-ASPabstracts. 156}

Background In 2013 the Palliative Medicine Department at Derby Hospitals launched an initiative within care homes with the aim of reducing unwanted hospitalisation and offering an alternative place of care for people approaching the end of their lives.

The Enhanced Beds Service consists of 10 beds within 5 Nursing Homes across a commissioning locality. They are available to patients who reach a crisis point, needing 24 hour care, where hospitalisation is undesirable. Day to day care is delivered by the Nursing Home staff, overseen by three specialist nurses who provide support and additional nursing care to patients and those important to them, as well as training and support to the Nursing Home staff.

Referrals are taken from all care providers including the hospital and community palliative care teams. Patients are assessed in their current location and, if admitted, are usually transferred within hours. A patient and carer experience survey and an audit are completed for all referrals. These measures monitor the service impact and direct improvements to the service.

Results In the last 4 years 466 patients have been transferred into an Enhanced Bed, $63 \%$ of which would have otherwise been admitted to hospital the same day - potentially saving 3500 acute bed days. Overall, 96\% of admissions avoid further hospitalisation. The experience survey reports a high level of satisfaction in the care received. Informal feedback from colleagues in health and social care is positive, with increasingly more complex patients being referred.

Recommendations The Enhanced Beds service has demonstrated measurable improvements in patient experience and outcomes. Future developments include a 'roaming bed' to increase choice of location.

\section{THE PRIDE OF WEST MIDLANDS PALLIATIVE CARE RESEARCH: WM CARES}

Hazel Coop, Mike Macfarlane, Matthew Doré, Clare Marlow, Sandra Prew, Derek Willis. WM CARES, NIHR, The Royal Wolverhampton NHS Trust, University of Chester

\subsection{6/bmjspcare-2018-ASPabstracts. 157}

Background Research is key for speciality development and higher medical training but there are well recognised barriers to participation in research within palliative care. The West Midlands Palliative Medicine Registrars recognised that lack of local structure and collaboration was contributing to trainees' difficulties in participating in research.

Methods The West Midlands Palliative Medicine Registrar group initiated a trainee-led collaborative, 'West Midlands Collaborative Actioning Research in End-of-life and Supportive Care' (WM CARES), which was launched in September 2016 with local stakeholder support. We hold monthly meetings at registrar training days, involving partners including the National Institute for Health Research (NIHR), local universities, palliative medicine consultants, speciality doctors and local providers of palliative care. We regularly update the wider palliative care community via newsletters, website and social media.

Results WM CARES has developed four research questions into working groups under consultant supervision. The WM CARES network enables larger, multi-site, high quality research which crosses the boundaries of any individual registrar's placement. To date, the group has presented six posters at national conferences and two journal articles are currently being prepared for publication. WM CARES has a mission to share local work, with collaboration being a central focus. This year the collaborative organised an inaugural conference, WM CARES PRIDE (Presentations in Research, Innovation, Development and Excellence) which showcased multidisciplinary research, audits and service developments from the region. There has been buy-in from the registrar body who recognise the opportunity to meet curriculum competences and explore research interests within a structured framework. Conclusion WM CARES is an innovative and collaborative trainee-led network conducting high-quality research and raising the profile of research. It enables and enthuses Palliative Medicine Registrars and the wider multidisciplinary team to learn about and be involved in research activities, which will ultimately improve patient care.

\section{INTEGRATED ONCOLOGY AND PALLIATIVE CARE: ANALYSIS OF A NEW SERVICE FOR CANCER PATIENTS}

Yukie Kano, Julie Nevin, Anna-Marie Stevens, Jayne Wood, Alison Kennett, Laila Kamal, Angela Halley, Joanne Droney. Royal Marsden NHS Foundation Trust

\subsection{6/bmjspcare-2018-ASPabstracts. 158}

Background There is growing evidence to support the benefits of early integrated palliative care (PC) for patients with advanced cancer. Within a tertiary referral cancer centre we started a new Integrated Symptom Control and PC service. The aim of this service is to proactively identify patients who would benefit from PC review and to offer earlier support. The aim of this study is to assess the feasibility and outcomes of this service.

Methods This pre-post design study was approved locally, and is part of a national programme to develop Integrated PC. Two tumour groups were selected due to their poor prognosis; renal cell cancer (RCC) and gynaecological cancer (GC). The study was conducted between October 2016 and September 2017. 316 patients (RCC 111/GC 205) were assessed as part of the new service. 286 of these patients (RCC 100/GC 186) were formally assessed for PC needs using the validated Integrated Palliative care Outcome Scale (IPOS). Descriptive statistical analysis was conducted. 